\title{
Airway pressure release ventilation in acute respiratory distress syndrome: children are not miniature adults
}

\author{
Vijai Williams ${ }^{1}$, Suresh Kumar Angurana', Muralidharan Jayashree ${ }^{1 *}$, Yongfang Zhou ${ }^{2}$ and Yan Kang ${ }^{2}$
}

(0) 2018 Springer-Verlag GmbH Germany, part of Springer Nature and ESICM

Initial correspondence from Drs. Williams, Angurana and Jayashree

Dear Editor,

In a recent article, Zhou et al. [1] found early application of airway pressure release ventilation (APRV) in adults with acute respiratory distress syndrome (ARDS) $(n=138)$ led to more ventilation-free days, better respiratory compliance, improved gas exchange, shorter ICU stays, and lower mortality (19.7\% vs. $34.3 \%)$ than in a low tidal volume (LTV) group. In this context, we would like to make a few important observations.

The LTV group had a higher incidence of ARDS caused by pneumonia and a higher proportion of patients $(50.7 \%$ vs. $32.4 \%$ ) with comorbidities (COPD, renal dysfunction and malignancy) and vasopressor requirement $(68.7 \%$ vs. 56.3\%). We believe these factors rather than the ventilation mode per se could have contributed to the poor outcomes in the LTV group. Furthermore, the higher incidence of tracheostomy (29.9\% vs. $12.7 \%)$ and need for sedation in the LTV compared with the APRV group also probably stemmed from the prolonged ventilation requirement. The seemingly lesser need for sedation and tracheostomy contrary to other published reports [2] in the latter is therefore debatable.

Can this be extrapolated to children? Literature on the usefulness of APRV in children with ARDS is limited. The first RCT on APRV in pediatric ARDS, by Lalgudi Ganesan et al. [3], reported contradictory findings. First, the APRV group had significantly higher mortality than

\footnotetext{
*Correspondence: mjshree@hotmail.com

${ }^{1}$ Department of Pediatrics, Advanced Pediatrics Centre, Postgraduate Institute of Medical Education and Research (PGIMER), Chandigarh, India
} Full author information is available at the end of the article the LTV group (53.8\% vs. $26.9 \%$ ), possibly because of the higher proportion of moderate and severe ARDS in the former than in the latter ( $96 \%$ vs. $73 \%$ ). Second, the similar sedation dose and duration in both ventilation groups could have worked against APRV by taking away the benefit of spontaneous breathing.

Since these two single-center studies on the use of APRV in ARDS are contradictory [1, 3], we need larger multicentric studies involving adults and children with a standardized protocol before APRV can be recommended across the board for the management of ARDS.

\section{Reply from Drs. Zhou and Kang}

We thank Drs. Williams, Angurana and Jayashree for their interest in our study and giving us an opportunity to clarify some questions.

First, the remarkable physiologic benefits of APRV have been reported in recent animal experiments. Nonetheless, data of patients with ARDS are still limited to small clinical trials with a broad variation in APRV settings, and the findings of these studies are controversial. The inherent problem was probably not the APRV mode, but might be the APRV methodology. At present, APRV has evolved into a highly sophisticated, physiology-driven, dynamic mechanical breath profile with precise settings. The traditional APRV settings were focused on Phigh, Thigh, Plow and Tlow.

Furthermore, in our study, to optimiz e the recruitment of alveoli gradually over time, while prevent alveoli overinflation and cyclic collapse, promote $\mathrm{CO}_{2}$ clearance and improve patient comfortable, first the settings of Phigh, Plow and Tlow were chosen according to pulmonary physiology mechanics (preventing alveolar overinflation and collapse, generating mandatory release volume); second, not Thigh but release frequency was directly selected, which was another major factor of mandatory 
mechanical release minute ventilation; third, APRV settings and dosages of analgesics and sedatives were jointly further titrated to achieve the target level of spontaneous breathing. Finally, we made a detailed titration of the APRV settings and weaning protocol. All of the above steps could affect the effect of APRV. Because this physiology-driven protocol is very complicated, first, our RT staff was well trained and used it skillfully, and then we conducted this single-center randomized controlled study.

Although there were more patients with co-existing chronic diseases in the LTV than in the APRV group, APRVwas independently associated with increased ventilator free days at day 28 after adjusting for the effect of chronic diseases on multivariable analysis. Also there was no statistically significant difference in the vasopressor requirement between both groups. APRV was associated with higher rates of successful extubation and lighter sedation. Lighter sedation could increase the number of ventilator-free days at day 28 ; however, APRV still significantly increased ventilator-free days at day 28 after correcting the sedation difference.

Finally, regarding the safety and efficacy of APRV in children, children of different ages usually need variable ventilation settings, especially the respiratory rate. Therefore, perhaps the methodology of APRV for children with ARDS needs further study in the future.

\author{
Author details \\ ${ }^{1}$ Department of Pediatrics, Advanced Pediatrics Centre, Postgraduate Institute \\ of Medical Education and Research (PGIMER), Chandigarh, India. ${ }^{2}$ Depart- \\ ment of Critical Care Medicine, West China Hospital of Sichuan University, \\ Chengdu 610041, China. \\ Funding \\ None. \\ Compliance with ethical standards

\section{Conflicts of interest} \\ The authors declare that they have no conflict of interests.
}

Received: 21 May 2018 Accepted: 26 July 2018

Published online: 6 August 2018

\section{References}

1. Zhou Y, Jin X, Lv Y et al (2017) Early application of airway pressure release ventilation may reduce the duration of mechanical ventilation in acute respiratory distress syndrome. Intensive Care Med 43:1648-1659. https:// doi.org/10.1007/s00134-017-4912-z

2. Bellani G, Laffey JG, Pham T et al (2016) The LUNG SAFE study: a presentation of the prevalence of ARDS according to the Berlin Definition! Crit Care 20(1):268. https://doi.org/10.1186/s13054-016-1443-x

3. Lalgudi Ganesan S, Jayashree M, Singhi SC, Bansal A (2018) Airway pressure release ventilation in pediatric acute respiratory distress syndrome: a randomized controlled trial. Am J Respir Crit Care Med. https://doi. org/10.1164/rccm.201705-09890C 